\title{
Entornos virtuales en la producción de textos en docentes de comunicación de la unidad de gestión educativa local Otuzco
}

\author{
Doris Aristela Rodríguez Hurtado \\ drodriguezh73@ucvvirtual.edu.pe \\ https://orcid.org/0000-0001-7502-6297 \\ Universidad César Vallejo Trujillo - Perú \\ Dulio Oseda Gago \\ dosedag@ucvvirtual.edu.pe \\ https://orcid.org/0000-0002-3136-6094 \\ Universidad César Vallejo Trujillo - Perú \\ Universidad César Vallejo
}

\section{RESUMEN}

El presente trabajo de investigación tuvo como objetivo determinar la influencia de los entornos virtuales en la producción de textos en los docentes del área de comunicación de la Unidad de Gestión Educativa Local de Otuzco en el año 2021. El tipo de investigación es básica, nivel correlacional causal y el diseño es no experimental: transversal descriptivo correlacional causal, teniendo como muestra a 69 docentes del área de comunicación de las diferentes instituciones educativas de la UGEL Otuzco a quienes se les administró dos cuestionarios en formularios de Google como instrumentos para el recojo de información de las dos variables. Los resultados obtenidos confirman que existe relación altamente significativa entre los Entornos Virtuales y Producción de Textos $r=, 544^{* *}$; (correlación moderada entre variables) para todo Sig. < $1 \%$. Se concluye que los entornos virtuales influyen en $26,8 \%$ en la producción de textos en los docentes de comunicación de la Unidad de Gestión Educativa Local de Otuzco en el año 2021.

Palabras clave: tecnológica; producción; creatividad; virtual; texto 


\title{
Virtual environments in the production of texts in communication teachers of the local educational management unit Otuzco
}

\begin{abstract}
The objective of this research work was to determine the influence of virtual environments in the production of texts in the teachers of the communication area of the Local Educational Management Unit of Otuzco in the year 2021. The type of research is basic, causal correlational level and the design is non-experimental: transversal descriptive correlational causal correlational, having as a sample 69 teachers in the area of communication of the different educational institutions of the UGEL Otuzco who were administered two questionnaires in Google forms as instruments for the collection of information of the two variables. The results obtained confirm that there is a highly significant relationship between Virtual Environments and Text Production $\mathrm{r}=, 544 * *$; (moderate correlation between variables) for all Sig. $<1 \%$. It is concluded that virtual environments influence $26.8 \%$ in the production of texts in communication teachers of the Local Educational Management Unit of Otuzco in the year 2021.
\end{abstract}

Keywords: technology; production; creativity; virtual; text

Artículo recibido: 18. Junio. 2021 Aceptado para publicación: 26. Julio. 2021 Correspondencia: aristelarodriguez@gmail.com Conflictos de Interés: Ninguna que declarar 


\section{INTRODUCCIÓN}

El escenario educativo en época de pandemia por COVID-19, ha expuesto la necesidad de los docentes en capacitación y actualización en entornos virtuales, herramientas tecnológicas y escritura digital para desarrollar con calidad la educación remota en los estudiantes de zonas urbanas y rurales. En este contexto, los profesores se convierten en agentes de cambio y para ello, requieren fortalecer sus habilidades y actitudes innovadoras, y asumir con emprendimiento pedagógico el uso de herramientas tecnológicas en la producción de textos impactantes y novedosos. En esta labor, los entornos virtuales se convierten en aliados para las propuestas de aprendizaje, contribuyendo a una educación capaz de dar respuesta a retos y oportunidades del siglo XXI. Para (Rodríguez et al., 2021) "La Competencia Digital Docente es cada vez más importante hacia un mejor uso de las tecnologías digitales en el aula”.

Para la producción de textos en entornos virtuales, se tiene en cuenta "las características del lenguaje, la comunicación y alfabetización en un contexto tecnológico, y la masificación del uso de dispositivos digitales conectados a internet” (Magad \& Bonnin, 2020). Sin embargo, los docentes tienen poco manejo de herramientas tecnológicas en la producción de textos, se suma a ello, la preocupación por los aprendizajes elementales de los estudiantes que evidencian dificultades en la organización de ideas coherentes y cohesionadas, así como en el uso del lenguaje. Según los resultados de la Evaluación Muestral de Escritura de alumnos del segundo grado de educación secundaria, aplicada por la Unidad de Medición de la Calidad (UMC), a nivel nacional, se tuvo que el $23,8 \%$ de estudiantes está en nivel Inicio y el $56 \%$ en nivel Proceso (Ministerio de Educación, 2018a). Frente a esta realidad, es propicio gestionar el potencial creativo de los docentes en la propuesta de estrategias y actividades para la producción creativa en entornos virtuales.

Además, para Ministerio de Educación (2016) "los docentes no diseñan actividades educativas interactivas aprovechando los entornos virtuales como viajar a otros lugares en tiempo y espacio, comprender cuentos o historias a través de simulaciones interactivas, grabar y compartir videos". Esta situación desafía a los profesores a adaptarse a los cambios de manera creativa y con soluciones originales y pertinentes proponiendo estrategias en la producción de textos como historietas, afiches, infografías, mapas mentales, presentaciones y videos con apoyo de herramientas 
tecnológicas y en los entornos virtuales. Según Reyes \& Avello-Martínez (2021) "el acceso y la masificación de la infraestructura digital ha provocado la necesidad de reconceptualizar la alfabetización digital como una forma de comprender cuáles son las habilidades que se necesita para poder participar en un mundo hiperconectado".

Los escenarios virtuales favorecen la implementación y desarrollo de estrategias de enseñanza mediante la innovación, propiciando impacto en el aprendizaje (MartínezPalmera et al., 2018); puesto que, su gran expectativa y sus infinitas aplicaciones han producido trascendentales implicancias en todos los ámbitos de la vida (Levano et al., 2019), proyectan efecto masivo y multiplicador en la educación, generando grandes cambios en la enseñanza de los profesores y al aprendizaje de los alumnos. Por ello, el uso de herramientas tecnológicas es indispensable en el entorno educativo. Para su integración en las aulas, es mediante aprendizajes significativos, resultado de experiencias vivenciales y contenidos reflexivos, que motivan a estudiantes y docentes a la producción de conocimiento (Ronald Hernández, 2017). Entonces, con mayor incidencia, los entornos virtuales favorecen la producción creativa.

El objetivo general de la investigación es determinar la influencia de los entornos virtuales en la producción de textos en los docentes del área de comunicación de la Unidad de Gestión Educativa Local de Otuzco en el año 2021. El planteamiento de la hipótesis respondió que los entornos virtuales influyen significativamente en la producción de textos en los docentes del área de comunicación de la Unidad de Gestión Educativa Local de Otuzco en el año 2021

Para la variable entornos virtuales, se tuvo en cuenta el Enfoque de Alfabetización Digital pues vivimos en una sociedad globalizada y en gran medida los entornos virtuales permiten a las personas interactuar. Para Martí et al. (2008) "Alfabetización digital se comenzó a utilizar en los años 90 para designar al conjunto de destrezas, conocimientos y actitudes que necesita una persona para poder desenvolverse funcionalmente dentro de la Sociedad de la Información". Para el Ministerio de Educación (2016) El propósito de la alfabetización digital, es desarrollar en los seres humanos habilidades para encontrar, comprender, socializar y construir información eficiente y participativamente, buscando el desempeño tecnológico e innovador en concordancia con la sociedad actual. (p. 215). 
La promoción de la competencia digital en los docentes y estudiantes involucra cuatro usos que deben ser controlados en la esfera digital. (a) Operativo, cuando aprenden a explorar las posibilidades máximas de algo en la web. (b) Semiótico, al interpretar diferentes códigos que mezclan lenguaje, imagen, video o sonido, y entienden cómo complementan la información textual. (c) Cívica, efectuando un uso responsable y autocrítico, conociendo los aspectos pragmáticos y éticos de la publicación en línea, y aceptando la responsabilidad de su desempeño. (d) Cultural, ya que necesitan mostrar respeto y una mentalidad abierta hacia diferentes prácticas culturales y puntos de vista dentro de la comunidad virtual del discurso de la biósfera (Pérez-Tornero, 2994 citado por Pascual, 2019).

La incursión de herramientas tecnológicas más sofisticadas y omnipresentes en educación, motiva a los profesores a planificar el uso pedagógico de las tecnologías, proponiendo colaboraciones en estos entornos y promoviendo investigación educativa en estrategias con la mediación de tecnologías (García \& Suárez, 2019). La incorporación de las TIC mediante la competencia transversal dentro del modelo “Aprovechamiento de las TIC" en amparo a la Ley General de Educación N²8044, busca aprendizajes óptimos con actividades motivadoras y desafiantes en entornos proporcionados por las TIC, sustentado en la alfabetización digital y la mediación interactiva (Ministerio de Educación, 2016).

En la actualidad, las TIC han tenido un crecimiento exponencial en la educación. El docente con una competencia digital adecuada aplica metodologías dinámicas y activas, generando cambios para convertirse en guía activo en la enseñanza y aprendizaje, mediante el desarrollo de métodos pedagógicos socioconstructivistas (González et al., 2014, Mon et al., 2014 y Cervera et al., 2016 citado por Moreno-Guerrero et al., 2020). "El modelo pedagógico constructivismo social utiliza las TIC como herramientas para el aprendizaje significativo a través de la combinación de experiencias, conexiones con el mundo real, retroalimentación e interacción" (Ferdig, 2007 citado por Ruiz \& Abella, 2011). En ese sentido, la producción de textos debe partir de experiencias cercanas y utilizando herramientas tecnológicas añadir diseños que impacten en el lector.

Puesto que, las TIC se han incorporado de manera rápida e ineludible a los escenarios educativos (Jaume et al., 2019); entonces, los entornos virtuales son los nuevos escenarios pedagógicos de la educación a distancia (Urquidi et al., 2019) y las 
tecnologías digitales contribuyen en el aprendizaje de manera permanente y en diferentes aspectos; incentivan y estructuran el desarrollo de habiliades y metodologías para aprender cada día (Jarvela, 2015 citado por Fosado et al., 2018). Las TIC se han convertido en un pilar principal del proceso de enseñanza aprendizaje, donde el estudiante las aprovecha para construir su aprendizaje significativo (Moreira, 2019). Por tales razones, surge, hoy más que nunca, la necesidad de utilizarlas en la producción de textos.

En ese sentido, se tiene en cuenta la personalización del entorno virtual, que consiste en adecuar la apariencia y funcionalidad de acuerdo con las actividades, valores, cultura y personalidad (Ministerio de Educación, 2016 p. 216). Es decir, se personaliza teniendo en cuenta que, los desafíos en el uso de las TIC responden a respectivos estilos, cultura diversa, pedagogía en e-learning y gestión del tiempo (Mustari et al., 2019). Además, los investigadores Pangrazio, Diosmío, \& Ledesma, (2020) manifiestan que la investigación educativa reconoce y define la necesidad de conceptualizaciones locales de alfabetizaciones digitales en sistemas educativos cada vez más globalizados.

Del mismo modo, las nuevas tecnologías han cambiado la forma de acceder a la información y la forma de interactuar; por ello, en los ambientes de aprendizaje, estos cambios se adaptan a la realidad del estudiante digital (Trejo, 2018). Con la gestión de información del entorno virtual, se organiza y sistematiza de manera ética y pertinente, tomando en cuenta sus tipos y niveles, así como la relevancia para las actividades (Ministerio de Educación, 2016 p. 216). Sin embargo, se debe comprender que la tecnología no facilita la tarea del docente, sino que la complementa y la transforma. La interacción docente-herramienta es clave en el proceso de aprendizaje. (Solé- Llussà et al., 2019). Por ello, saber gestionar la información en beneficio de la educación, es fundamental en estos entornos.

Respecto a la interacción en los entornos virtuales, consiste en realizar actividades en conjunto y construir vínculos coherentes según valores y contexto sociocultural (Ministerio de Educación, 2016 p. 216). Herramientas tecnológicas como el blog, permiten a estudiantes y docentes interactuar de manera activa mediante la redacción y publicación de textos. Fortalece la competencia digital, propicia el debate y la construcción de conocimientos sin barreras espaciales y temporales (Marín et al., 2020); además, facilita que los estudiantes incrementen sus técnicas de innovación en clases 
entretenidas (Mustari et al., 2019), aportando interactividad y contribuyendo al trabajo cooperativo (Gutierrez, 2017). Por todo ello, el blog es un entorno virtual favorable para promover la interacción y la producción de textos.

Referente a la creación de objetos virtuales, es el resultado de un proceso de mejoras sucesivas y retroalimentación desde el contexto escolar y en la vida cotidiana para construir materiales digitales con diversos propósitos (Ministerio de Educación, 2016 p. 216). Las TIC ponen a disposición un grandioso número de herramientas digitales y escenarios para el campo educativo donde los OVA (Virtual Learning Objets) se adaptan a diversos contextos tecnológicos y potencializan las competencias de los estudiantes. Son de utilidad para una educación innovadora y pertinente. (MartínezPalmera et al., 2018). Los OVA permiten trabajar temas e interactuar con el público objetivo, en relación con variadas áreas del conocimiento.

En este escenario, una atrayente plataforma diseñada para crear cómics o historietas en línea es Pixton. Mediante esta herramienta se crean materiales visuales atractivos para la participación de los estudiantes, por lo tanto, facilita la producción de diálogos utilizando los contenidos de las áreas en relación con sus estilos y predisposiciones de aprendizaje (Cabrera et al., 2018). PowToon es otra herramienta para crear OVA. La propuesta de estrategias didácticas basadas en esta herramienta, propician el aprendizaje creativo de los estudiantes, generando reflexión, análisis, comprensión. (Arroyo-Carrera et al., 2020). Asimismo, PowToon fomenta el aprendizaje colaborativo para favorecer un desarrollo y fortalecimiento en alfabetización digital. En este contexto tecnológico, las aplicaciones novedosas que facilitan la producción de textos de manera creativa y crítica, entre otras también son Canva y Mindomo.

Figura 1: Herramientas tecnológicas en la producción de textos

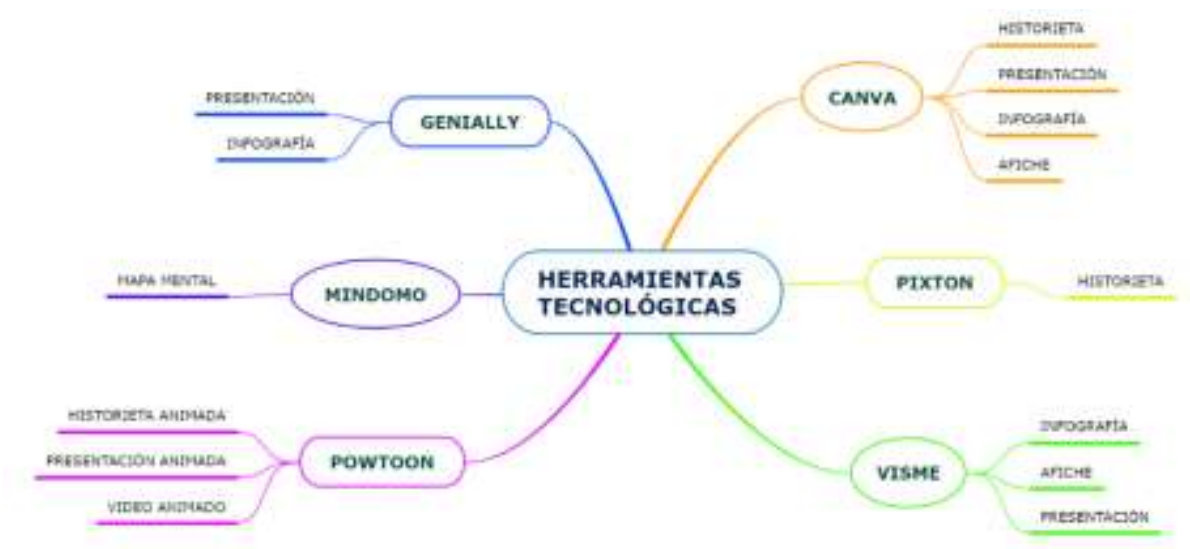

Fuente: Elaborado por la investigadora en Mindomo. 
El marco teórico y metodológico que orienta el desarrollo de la producción de textos, es el enfoque comunicativo; puesto que, se usa el lenguaje para comunicarse con los demás, comprendiendo y produciendo textos orales y escritos de distinto tipo textual, formato y género discursivo, con propósitos variados y en distintos soportes, como impresos, audiovisuales, digitales, entre otros. Además, considera las prácticas sociales del lenguaje ya que la comunicación se produce mediante la interacción de las personas en la vida social y cultural. Asimismo, enfatiza lo sociocultural; dado que, los usos y prácticas del lenguaje se dan en contextos socioculturales determinados. Es decir, los lenguajes orales y escritos adoptan características propias de cada contexto (Ministerio de Educación, 2016).

Definiendo la palabra escribir, tenemos según (Niño, 2011 p. 156):

"Escribir es un acto de creación mental en el que un sujeto escritor, con un propósito comunicativo, concibe y elabora un significado global, y lo comunica a un lector mediante la composición de un texto, valiéndose del código de la lengua escrita"

En otras palabras, para producir un texto, el escritor debe tener un propósito claro y mediante la creación intelectual, imagina, inventa y procesa un significado total y lo comunica al lector mediante el uso del lenguaje escrito.

"La competencia Escribe diversos tipos de textos en su lengua materna comprende usar el lenguaje escrito para construir sentidos o mensajes y comunicarlos mediante el texto". (Ministerio de Educación, 2016). Hoy en día, la educación requiere de profesionales competentes, que resuelvan "procesos complejos de desempeño con idoneidad en un determinado contexto y con responsabilidad" (Tobón, 2006). La producción de textos comprende un proceso sistemático y reflexivo que permite la combinación de las capacidades: adecuación a la situación; organización y desarrollo de las ideas mediante diferentes estrategias y técnicas para que sean coherentes y estén debidamente cohesionadas; uso del lenguaje mediante la utilización de un conjunto de convenciones de la escritura, y la reflexión y revisión que debe ser constante para mejorar los escritos (Ministerio de Educación, 2016).

Según, (Cassany, 2010) La corriente sobre procesos de composición del escrito son una línea de investigación psicolingüística y un movimiento de renovación de la enseñanza 
de la redacción. Siendo su campo de acción es el proceso de composición o de escritura. A continuación, se cita 3 implicaciones de esta corriente:

"No hay una única manera de escribir, sino que cada cual tiene que encontrar su estilo personal de composición".

"Escribir es un proceso de elaboración de ideas, además de una tarea lingüística de redacción. El escritor tiene que saber trabajar con las ideas tanto como con las palabras".

"Escribir es un instrumento epistemológico de aprendizaje. Escribiendo se aprende y podemos usar la escritura para comprender mejor cualquier tema”.

Se ha tenido en cuenta aspectos del modelo de producción de textos de Beaugrande y Dressler (1981) tales como:

a) la intencionalidad (actitud del escritor) proyectada hacia la orientación de la actividad interpretativa para la consecución de una meta; b) la aceptabilidad (actitud del receptor) que realiza el interlocutor de la cohesión, coherencia e intencionalidad del texto como relevante; c) la situacionalidad (relevancia) o pertinencia del texto en un contexto de interacción; d) la intertextualidad (relación entre textos) o interpretación y comprensión del texto a partir de la información recibida de otros textos anteriores, y e) la informatividad o novedad y relevancia del significado del texto, citado por (Álvarez \& Ramírez, 2006).

Figura 2: Recreación del modelo de producción de textos de Beaugrande y Dressler (1981)

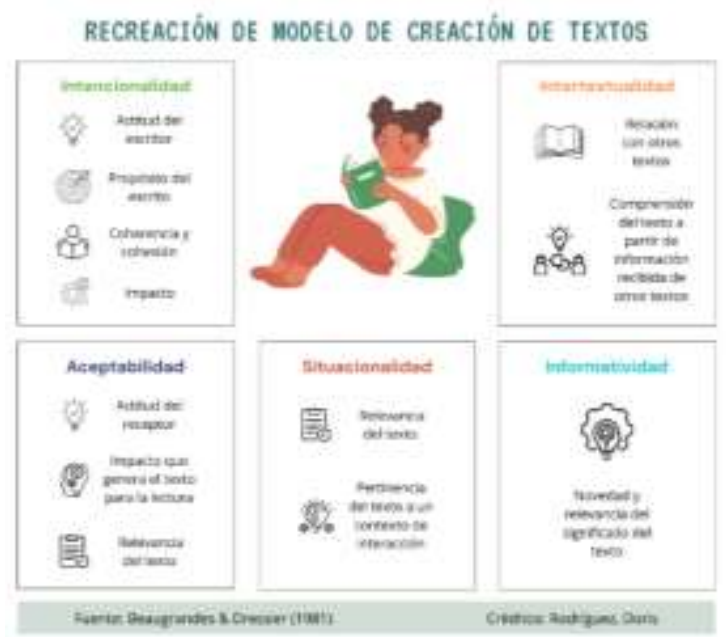

Fuente: elaborado por la investigadora en plantillas de Canva. 
Según lo citado por los mismos autores se tiene los principios regulativos de la comunicación textual:

a) eficacia de la información para facilitar el procesamiento textual o comprensión, b) efectividad o nivel de impacto significativo que el texto provoca en los lectores, y c) adecuación para determinar la consistencia entre el texto y las normas de textualización y se procese la información con facilidad. Además, de estos principios, se sigue el proceso de acceso a la información, planificación, redacción y reflexión del texto.

Como práctica social, la escritura motiva a construir conocimiento y usar estéticamente el lenguaje. Es una forma de vivir las costumbres sociales y culturales de las comunidades y ofrecer la posibilidad de interactuar con los demás a través de los escritos donde se crea y recrea mundos, personajes, se comunica emociones, sentimientos y se genera impacto en el lector (Ministerio de Educación, 2016). Debido a "la relevancia innegable que tiene la escritura en la vida social, académica y secular de los ciudadanos, su adquisición, dominio y puesta en práctica, se relaciona directamente con la producción científica y académica de un país.”(Piñeros et al., 2018 p. 176).

La escritura influye en lo social, cultural y científico de los pueblos. Los textos son producto del trabajo creativo realizado en el contexto del aula por los sujetos (Pastor, 2018); por ello, el docente a cargo de la enseñanza de la escritura explora el contexto, material sobre didáctica, procesos y técnicas de escritura. En ese sentido, cada día fortalece su práctica pedagógica a partir de la escritura de sus propios textos. Puesto que, "la enseñanza debe ser guiada y modelada, y el profesor de escritura, fomentar la aparición de necesidades de aprendizaje e instancias de participación social e individual" (Navarro, 2019).

Los contenidos visuales apoyados en elementos gráficos permiten la fácil comprensión de la información. Así, encontramos textos discontinuos en medios físicos, como representaciones en diarios, catálogo, mapa, itinerario; aviso publicitario.(Ministerio de Educación, 2018b). Mediante los textos se expresa las vivencias, las emociones, opiniones, puntos de vista, creencias y saberes. En su producción se resalta el juicio independiente, por ello, se tiene en cuenta que los textos son herramientas para la construcción de nuevos conceptos buscando la expresión de un discurso propio (Arango \& Murillo, 2018). A continuación se presenta cuatro textos discontinuos: 
La infografía se elabora de manera estructurada y organizada con textos, ilustraciones, estadísticas y otras gráficas con información científica, académica, etc., y con flexibilidad en el formato. La encontramos en periódicos, televisión, internet, libros, artículos científicos, informes empresariales, redes sociales, museos y centros de aprendizaje interactivo (Ruiz, 2020 p. 401). Contextualiza la información mediante un mapa, clarifica los datos con un gráfico de barras, recopila información en una tabla o una ilustración para hacer una página más ligera visualmente. Si es en línea, se integra la hipertextualidad, interactividad o multimedialidad. (Ivars-Nicolás, 2019) (p. 384). La infografía es un recurso educativo y didáctico que facilita la apropiación de nuevos conocimientos, se adapta a cualquier tema, campo de conocimiento y nivel académico. (Arenas-Arredondo et al., 2021 p. 270). Su presentación genera impacto para la lectura. El mapa mental, que es un organizador gráfico del conocimiento, facilita la organización y comprensión del concepto complejo y su desagregación en unidades más pequeñas. El gráfico contribuye a la priorización de los conceptos, de lo general a lo específico, La información se presenta en una estructura coherente, permitiendo un recorrido visual lógico a través de la relación secuencial de los conceptos. Ayuda a visualizar la información de forma integral, para su construcción, se utilizan flechas y fuentes de diferentes tamaños de letra (Blandi et al., 2021). Es un organizador visual armónicos con semejanzas a la neurona cerebral (Novoa, 2018). Para su elaboración se requiere de creatividad e información confiable, significatividad y armonía en gráficos y textos.

La historieta, según Altarriba (2011) “es el arte de contar historias en imágenes, pueden ser de humor, aventura, fantasía, poesía, historia, periodismo o inclasificables. Todas ellas narradas siguiendo un código, una gramática pictórica, una retórica escriptoicónica, una combinación léxico-gráfico, que las dota de originalidad". La historieta se ha convertido en un medio de innovación, de lectura sorpresiva y altamente gratificante. Sobresale por ser creativa, trascendente, experimental, testimonial o satírica, y es leída por un público abierto a nuevas formas narrativas. Para su diseño prevalece la forma narrativa basada en la imagen secuencial (Conde, 2019). Son novedosas y singulares que impactan al lector.

El afiche, en la elaboración se combinan dos lenguajes: el de la imagen y el del texto, la imagen muestra y el texto explica (Copello, 2004). El afiche publicitario considera 
como elementos principales la imagen y el eslogan convincente y breve aludiendo a aquello que el consumidor obtendrá al adquirir el producto o servicio (Giraldo, 2021).

\section{MÉTODO}

La investigación fue de tipo básica orientada a incrementar los conocimientos científicos de algunos aspectos de la realidad (Oseda et al., 2018 p. 167). Se desarrolló bajo el enfoque cuantitativo, se empeló la estadística para dar respuesta al problema planteado (Hernández et al., 2014). El nivel de investigación fue descriptivo correlacional, para establecer el grado de relación o asociación no causal entre las dos variables. Primero se midieron las variables y luego, la correlación, mediante pruebas de hipótesis correlacionales y la aplicación de técnicas estadísticas (Oseda et al., 2018 p. 172).

El diseño de investigación fue el no experimental transversal debido a que no hubo intervención o manipulación de las variables y la recolección de datos, y su estudio se realizó en un tiempo único, (Oseda et al., 2018), siendo el descriptivo correlacional causal, toda vez que se propuso que en una misma muestra específica, se midan las dos variables de estudio: entornos virtuales y producción de textos, luego se compararon o correlacionaron estadísticamente, mediante un coeficiente de correlación.

Con el siguiente esquema

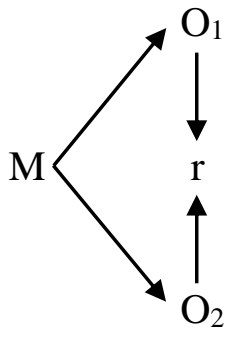

Donde: $M=$ Muestra.

O1 = Observación de la variable 1 (entornos virtuales).

$\mathrm{O} 2$ = Observación de la variable 2 (producción de textos).

$\mathrm{r}=$ relación entre las dos variables.

La muestra estuvo conformada por 69 docentes del área de comunicación de las instituciones educativas de la UGEL Otuzco, en el año 2021.

Se trabajó con la técnica de la encuesta, teniendo como instrumentos para la recolección de datos dos cuestionarios estandarizados y creados en los formularios de Google, uno 
por cada variable. Para cada instrumento se elaboró ficha técnica; por lo tanto, se procedió a la validación mediante juicio de expertos de amplia trayectoria.

El cuestionario de la variable entornos virtuales se elaboró teniendo en cuenta las 4 dimensiones y los 6 indicadores. Estuvo constituido por 16 ítems, 4 para cada dimensión. El cuestionario de la variable producción de textos se diseñó en base a las 4 dimensiones y 4 indicadores con un total de 20 ítems, 5 para cada dimensión.

De acuerdo al enfoque cuantitativo, se elaboró la base de datos para la variable entornos virtuales y para producción de textos. Esta data se obtuvo a partir de las respuestas de los cuestionarios aplicados de manera virtual. En el procesamiento de datos se empleó el programa Microsoft Excel y SPSS, versión 25, en la creación de tablas a partir del análisis. Para la presentación de resultados se elaboraron tablas de frecuencia para resumir información de las variables de estudio. Para la prueba de hipótesis se realizó con Rho de Sperman y con la regresión múltiple debido a las escalas ordinales utilizadas. Se realizó la correlación entre variables y dimensiones. Se discutió los resultados encontrados y se arribó a las conclusiones.

\section{RESULTADOS Y DISCUSIÓN}

Los resultados de los datos obtenidos de las variables de estudio, aplicados a la muestra, se visualizan en la siguiente tabla de frecuencia y porcentaje.

Tabla 1. Niveles de las Variables: Entornos Virtuales y Producción de Textos

\begin{tabular}{l|cccc}
\hline \multirow{2}{*}{ Niveles } & \multicolumn{2}{c}{ Entornos virtuales } & \multicolumn{2}{c}{ Producción de textos } \\
\cline { 2 - 5 } & frecuencia & $\%$ & 0 & 0,0 \\
\hline Bajo & 0 & 0.0 & 39 & 56,5 \\
Medio & 24 & 34,8 & 30 & 43,5 \\
Alto & 45 & 65,2 & 69 & 100,0 \\
\hline Total & 69 & 100,0 & 6 \\
\hline
\end{tabular}

Fuente. Data de Entornos Virtuales y Producción de Textos

\section{Interpretación}

Después de haber aplicado el instrumento de la variable 1 Entornos virtuales a los 69 docentes del área de Comunicación de la Unidad de Gestión Educativa Local de Otuzco - 2021, se tiene que 45 docentes que representa el 65,2\% estuvieron en el nivel alto, luego 24 docentes que es el $34,87 \%$ en el nivel medio y en el nivel bajo no se tuvo a 
ningún docente. Como se puede apreciar la mayor cantidad está en el nivel alto, el cual es positivo.

Respecto a la aplicación del instrumento de la variable 2 producción de textos, se tiene que 39 docentes que representa el 56,5\% estuvieron en el nivel medio, luego 30 docentes que es el 43,5\% en el nivel alto y en el nivel bajo no se tuvo a ningún docente. Como se observa, la mayor cantidad está en el nivel medio, el cual es positivo.

Hipótesis: Existe relación significativa entre entornos virtuales y producción de textos en los docentes del área de Comunicación de la Unidad de Gestión Educativa Local de Otuzco - 2021.

Nivel de significancia o riesgo: $\alpha=0,05$.

Tabla 2. Coeficiente de correlación

\begin{tabular}{|c|c|c|c|c|}
\hline \multicolumn{3}{|c|}{ Correlación } & $\begin{array}{l}\text { Entornos } \\
\text { virtuales }\end{array}$ & $\begin{array}{c}\text { Producción } \\
\text { de textos }\end{array}$ \\
\hline \multirow{6}{*}{$\begin{array}{l}\text { Rho de } \\
\text { Spearman }\end{array}$} & \multirow{3}{*}{$\begin{array}{l}\text { Entornos } \\
\text { virtuales }\end{array}$} & Coeficiente de correlación & 1,000 &, $544^{* *}$ \\
\hline & & Sig. (bilateral) & . & ,000 \\
\hline & & $\mathrm{N}$ & 69 & 69 \\
\hline & \multirow{3}{*}{$\begin{array}{l}\text { Producción } \\
\text { de textos }\end{array}$} & Coeficiente de correlación &, $544^{* *}$ & 1,000 \\
\hline & & Sig. (bilateral) & 000 & $\cdot$ \\
\hline & & $\mathrm{N}$ & 69 & 69 \\
\hline
\end{tabular}

Fuente: Data de Entornos Virtuales y Producción de Textos

Decisión estadística: Puesto que (p-valor: $0,000<0,010$ y rho $=0,544$, en consecuencia, se acepta la hipótesis.

Conclusión estadística: Se concluye que existe una relación altamente significativa entre entornos virtuales y producción de textos en los docentes del área de Comunicación de la Unidad de Gestión Educativa Local de Otuzco - 2021, expresada en $r=, 544^{* *}$; (correlación moderada entre entornos virtuales con producción de textos) para todo Sig. $\mathrm{p}<1 \%$.

Estadísticamente se tiene que (p-valor: $0,000<0,010$ ), en consecuencia, se acepta la hipótesis, se concluye que existe una relación altamente significativa entre entornos virtuales y producción de textos en los docentes del área de Comunicación de la Unidad de Gestión Educativa Local de Otuzco - 2021. 
Tabla 3. Influencia de los Entornos Virtuales en la Producción de Textos

\begin{tabular}{lc|ccc}
\hline Modelo & R & R cuadrado & R cuadrado ajustado & $\begin{array}{c}\text { Error estándar } \\
\text { de la estimación }\end{array}$ \\
\hline 1 &, $518^{\mathrm{a}}$ &, 268 &, 257 & 4,990 \\
\hline
\end{tabular}

a. Predictores: (Constante), Entornos Virtuales

Fuente: Data de Entornos Virtuales y Producción de Textos y Dimensiones

\section{Interpretación}

Los entornos virtuales influyen en $26,8 \%$ en la producción de textos; el $73,2 \%$ es influida por otras variables.

Como se puede apreciar, se cumple la hipótesis planteada en la investigación que los entornos virtuales influyen significativamente en la producción de textos; sin embargo, en la variable 2, también influyen otros factores o variables.

Tabla 4. Influencia de los Entornos Virtuales en las dimensiones de la variable producción de textos

\begin{tabular}{l|c|c|c|c|}
\hline \multicolumn{1}{|c|}{ Modelo } & $\mathbf{R}$ & R cuadrado & $\begin{array}{c}\text { R cuadrado } \\
\text { ajustado }\end{array}$ & $\begin{array}{c}\text { Error estándar de } \\
\text { la estimación }\end{array}$ \\
\hline Infografía &, $683^{\mathrm{a}}$ &, 467 &, 459 & 1,275 \\
\hline Mapa mental &, $445^{\mathrm{a}}$ &, 198 &, 186 & 1,443 \\
\hline $\begin{array}{l}\text { Historieta } \\
\text { Afiche }\end{array}$ &, $371^{\mathrm{a}}$ &, 138 &, 125 & 1,518 \\
\hline
\end{tabular}

a. Predictores: (Constante), Entornos Virtuales

Fuente: Data de Entornos Virtuales y Producción de Textos y Dimensiones

\section{Interpretación}

Los entornos virtuales influyen en 46,7\% en la producción de infografías; en 19,8\% en la producción de mapas mentales; en 13,8\% en la producción de historietas, y en 9,4\% en la producción de afiches.

De igual forma, las hipótesis específicas se cumplen al demostrase que los entornos virtuales influyen de manera significativa en las dimensiones de infografía, mapa mental, historieta y afiche. En menor medida se evidencia que hay influencia en la dimensión 4.

Estos resultados se discuten con Torres (2018) que en su tesis de posgrado: "Uso de la Tecnología de la Información y Comunicación (TIC) y las competencias comunicativas en estudiantes de los primeros ciclos del Instituto Tecnológico TECSUP - 2018”. 
Universidad Nacional de Educación Enrique Guzmán y Valle, concluye que el uso de las Tecnologías de información y comunicación (TIC) influyen significativamente en el desarrollo de la producción de textos en estudiantes de los primeros ciclos del Instituto Tecnológico TECSUP ( $\mathrm{p}=0,000$ ). El 95\% de estudiantes del grupo experimental alcanzó el nivel Regular en la prueba de salida. Muy similar a nuestros resultados. Además, se ajusta de manera total a la relación que existe entre las dos variables de estudio.

Del mismo modo, Demarini (2017) en su tesis: "Efectos del uso de las TIC en la producción de textos argumentativos en estudiantes del 5to de secundaria de la Institución Educativa N 1201 Paul Harris - La Victoria - 2017”. Universidad Nacional de Educación Enrique Guzmán y Valle, muestra mediante la prueba U de Mann Whitney aplicada al Grupo Control y Experimental en la post prueba, el nivel de significancia que es menor a $0,05(0,000<0,05)$. Concluye que, existe evidencia estadística para afirmar que el uso de las TIC produce efectos significativos en la producción de textos argumentativos en estudiantes de quinto de secundaria de la Institución Educativa N¹201 Paul Harris, La Victoria, 2017. Muy similar a nuestros resultados que tiene significancia de $0,00, \mathrm{p}=0,000<0,05$ y Rho de Spearman $=$ , $544^{* *}$.

Asimismo, se realiza la discusión con Domingo-Coscollola et al., (2019) En su trabajo de investigación, concluye que: Los recursos digitales se usaron para enseñar y aprender. El dominio de la Competencia Digital Docente del profesorado universitario es deficiente; generalmente menor, en la Competencia Docente Metodológica que en la Competencia Docente Instrumental. La alfabetización digital del alumnado es una necesidad y para posibilitarla, se destacan entornos que fomenten el aprendizaje colaborativo mediante las TIC. Se discute con los resultados de nuestra investigación debido al fomento de aprendizajes mediante las TIC utilizando los entornos virtuales y existiendo la necesidad la alfabetización digital en los docentes. En lo relacionado a destacar la ética y civismo digital en la acción educativa, el uso responsable, seguro y saludable de estas tecnologías; así como el respeto a la propiedad intelectual, se discute que la coincidencia es total; puesto que en los ítems del instrumento se mencionan la gestión de información con fuentes confiables respetando el derecho de autor. Este trabajo tiene mayor coincidencia en la variable 1, entornos virtuales y menor relación en 
producción de textos; sin embargo, ambos estudios destacan que las tecnologías digitales adquieren un papel integral en el aprendizaje a lo largo de la vida.

\section{CONCLUSIONES O CONSIDERACIONES FINALES}

1) Los entornos virtuales influyen de manera significativa en $26,8 \%$ en la producción de textos en los docentes del área de comunicación de la Unidad de Gestión Educativa Local de Otuzco 2021.

2) Los entornos virtuales influyen de manera significativa en la producción de infografías $(46,7 \%)$ en los docentes del área de comunicación de la Unidad de Gestión Educativa Local de Otuzco 2021.

3) Los entornos virtuales influyen en nivel medio en la producción de mapas mentales $(19,8 \%)$ en los docentes del área de comunicación de la Unidad de Gestión Educativa Local de Otuzco 2021.

4) Los entornos virtuales influyen en nivel medio en la producción de historietas $(13,8 \%)$ en los docentes del área de comunicación de la Unidad de Gestión Educativa Local de Otuzco 2021.

5) Los entornos virtuales influyen en nivel medio en la producción de afiches $(9,4 \%)$ en los docentes del área de comunicación de la Unidad de Gestión Educativa Local de Otuzco 2021.

\section{REFERENCIAS BILIOGRAFICAS}

Altarriba, A. (2011). Introducción sobre el origen, evolución, límites y otros debates teóricos en torno a la historieta. Arbor, 187(EXTRA 2), 9-14. https://doi.org/10.3989/arbor.2011.2extran2111

Álvarez, T., \& Ramírez, R. (2006). Teorías o modelos de producción de textos en la enseñanza y el aprendizaje de la escritura. Didáctica (Lengua y Literatura), January 2006. https://doi.org/10.5209/rev

Arenas-Arredondo, A., Harringhton-Martínez, M., Varguillas-Carmona, C., \& GallardoVarguillas, D. (2021). Infográficos : uso na educação. Dominio de Las Ciencias, 7, 261-284. https://doi.org/10.23857/dc.v7i1.1640

Balladares-Burgos, J. (2018). Diseño pedagógico de la educación digital para la formación del profesorado Instructional design of digital education for teacher training. Revista Latinoamericana de Tecnología Educativa, 17(1), 41-60. https://doi.org/10.17398/1695-288X.17.1.41 
Blandi, M., Gargoloff, N., Iermanó, M., Paleologos, M., \& Sarandón, S. (2021). The mental map as an instrument to define indicators in complex systems: an application on the environmental knowledge of local horticultural farmers. Revista de Economia e Sociologia Rural, 60(1), 1-17. https://doi.org/10.1590/1806-9479.2021.233521

Cabrera, P., Castillo, L., González, P., Quiñónez, A., \& Ochoa, C. (2018). The impact of using Pixton for teaching grammar and vocabulary in the EFL Ecuadorian context. Teaching English with Technology, 18(1), 53-76.

Cassany, D. (2010). La cocina de la escritura (A. S.A. (ed.); Decimosépt).

Conde, J. (2019). Del cómic a la novela gráfica: mutaciones editoriales de la historieta colombiana en el siglo XXI. Mitologías Hoy, 20(0), 61-77.

Copello, M. (2004). El afiche como paradigma de la comunicación. Huellas, 112-116.

Cukierman, U., Palmieri, J., \& de Lima, D. G. (2020). An experience of educational innovation in engineering education: 10 years promoting the approach by competences in the undergrad. Proceedings of the LACCEI International MultiConference for Engineering, Education and Technology. https://doi.org/10.18687/LACCEI2020.1.1.619

Domingo-Coscollola, M., Bosco-Paniagua, A., Carrasco-Segovia, S., \& SánchezValero, J.-A. (2019). Fomentando la competencia digital docente en la universidad: Percepción de estudiantes y docentes. Revista de Investigación Educativa, 38(1), 167-182. https://doi.org/10.6018/rie.340551

Fosado, R., Martínez, A., Hernández, N., \& Ávila, R. (2018). El portafolio virtual como una herramienta transversal de planeación y evaluación del aprendizaje autónomo para el desarrollo sustentable. Revista Iberoamericana Para La $\begin{array}{llllll}\text { Investigación } & y & E l & \text { Desarrollo } & \text { Educativo, } & 8 .\end{array}$ https://doi.org/10.23913/ride.v8i16.338

García, M., \& Suárez, C. (2019). Estado de la investigación sobre la colaboración en Entornos Virtuales de Aprendizaje. PIXEL BIT Revista de Medios y Educación, $169-191$.

Giraldo, D. (2021). Producción de textos argumentativos publicitarios sobre promoción lectora. Propuesta en la formación inicial de docentes Production of Argumentative Advertising Texts on Reading Promotion: Proposal for Initial 
Teacher Training Produção de textos publici. Sophia, 17(1), 1-12. https://doi.org/10.18634/sophiaj.17v.1i.1103

Hernández, Roberto, Fernández, C., \& Baptista, M. (2014). Metodología de la Investigación (M.-H. E. S.A. (ed.); sexta).

Hernández, Ronald. (2017). The incorporation and use of ICT in early childhood education. A study on infrastructure, teaching methods and teacher training in Andalusia. Propositos y Representaciones, 5(52), 325-347. https://doi.org/10.20511/pyr2017.v5n1.149

Ivars-Nicolás, B. (2019). La infografía periodística en España: definición y tipología de uso. Estudios Sobre El Mensaje Periodístico, 25(1), 283-302. https://doi.org/10.5209/esmp.63729

Jaume, M., Perales, F., Negre-Bennasar, F., \& Fontanet, G. (2019). Accessible web design and documentation in university learning. Revista de Educacion a Distancia, 19(60). https://doi.org/10.6018/red/60/06

Levano, L., Diaz, S., Guillen, P., Tello, S., Herrera, N., \& Collantes, Z. (2019). Digital competences in education. Propositos y Representaciones, 7(2), 569-588.

Magad, C., \& Bonnin, J. E. (2020). Escritura digital y colaborativa: una práctica discursiva multifacética. Estado del arte y perspectivas para el futuro. Quintú Quimün. Revista de Lingüística, O(4), 028.

Marín, V., Sampedro, B., Muñoz, J., \& Salcedo, P. (2020). The blog in the training of education professionals. Revista Electronica Interuniversitaria de Formacion Del Profesorado, 23(2), 113-126. https://doi.org/10.6018/reifop.414061

Martínez-Palmera, O., Combita-Niño, H., \& De-La-Hoz-Franco, E. (2018). Mediation of virtual learning objects in the development of mathematical competences in engineering students. Formacion Universitaria, 11(6), 63-74. https://doi.org/10.4067/S0718-50062018000600063

Ministerio de Educación. (2016). Programa Curricular de Educacion Secundaria.

Ministerio de Educación. (2018a). ¿Qué logran nuestros estudiantes en escritura?

Ministerio de Educación. (2018b). Marco de evaluación de la competencia lectora de PISA 2018.

Moreira, P. (2019). Las Tics en el aprendizaje significativo y su rol en el desarrollo cognitivo de los adolescentes. ReHuSo: Revista de Ciencias Humanísticas y 
Sociales.

$e-I S S N$

$2550-6587$.

$U R L:$

Www.Revistas.Utm.Edu.Ec/Index.Php/Rehuso,

$4(2)$

1. https://doi.org/10.33936/rehuso.v4i2.1845

Moreno-Guerrero, A., Miaja-Chippirraz, N., Bueno-Pedrero, A., \& Borrego-Otero, L. (2020). El área de información y alfabetización informacional de la competencia digital docente. Revista Electrónica Educare, 24(3), 1-16. https://doi.org/10.15359/ree.24-3.25

Mustari, M., Hoya, A. L., Akmansyah, M., Diani, R., \& Asyhari, A. (2019). Development of E-Learning Based Blogs on Global Warming Subject. Journal of Physics: Conference Series, 1155(1). https://doi.org/10.1088/17426596/1155/1/012036

Niño, V. (2011). Competencias en la comunicación: Hacia las prácticas del discurso (Ecoe (ed.); Tercera).

Novoa, P. (2018). El Mapa Mental Armónico en la comprensión de textos narrativos en estudiantes universitarios. In Propósitos y Representaciones.

Oseda, D., Santacruz, A., Zevallos, L., Sangama, J., Cosme, L., \& Mendievel, R. (2018). Fundamentos de la Investigación Científica (S. Gráficas (ed.)).

Pascual, D. (2019). Learning English With Travel Blogs: A Genre-Based ProcessWriting Teaching Proposal Aprendiendo inglés con blogs de viajes: una propuesta basada. Profile Issues in Teachers' Professional Development. https://doi.org/10.15446/profile.v21n1.71253

Pastor, M. D. G. (2018). Textos de identidad digitales: Una valiosa herramienta para el estudio de la construcción de la identidad y el aprendizaje lingüístico en ILE. Revista Signos, 51(96), 24-44. https://doi.org/10.4067/S071809342018000100024

Piñeros, M., Orjuela, D., \& Torres, A. (2018). A Critical Insight into the Writing Practices of Fifth Graders. Cuadernos de Lingüística Hispánica, 31, 190.

Reyes, C. E. G., \& Avello-Martínez, R. (2021). Digital literacy in education. Systematic review of scientific production in Scopus. Revista de Educacion a Distancia, 21(66). https://doi.org/10.6018/RED.444751

Rodríguez, M. U., Cantabrana, J. L. L., \& Cervera, M. G. (2021). Validation of a tool for self-evaluating teacher digital competence. Educacion XX1, 24(1), 353-373. 
https://doi.org/10.5944/educXX1.27080

Ruiz, Maritza. (2020). The journalistic digital infographic and its contribution to the school $\quad$ Kess. $\quad$ Kes, 395-435. https://doi.org/10.17151/kepes.2020.17.21.14

Ruiz, Mónica, \& Abella, V. (2011). Creación de un blog educativo como herramienta TIC e instrumento TAC en el ámbito universitario. Teoría de La Educación. Educación y Cultura En La Sociedad de La Información, 12, 53-70.

Solé- Llussà, A., Aguilar-Camaño, D., \& Ibáñez-Plana, M. (2019). Las ayudas en indagaciones científicas escolares mediadas por herramientas tecnológicas. Investigaciones de la última década. Digital Education Review, 36, 223-242. https://doi.org/10.1344/der.2019.36.223-242

Trejo, H. (2018). Herramientas tecnológicas para el diseño de materiales visuales en entornos educativos. $\quad$ Sincronía, $617-669$. https://doi.org/10.32870/sincronia.axxii.n74.30b18

Urquidi, A., Calabor, M., \& Tamarit, C. (2019). Entornos virtuales de aprendizaje: modelo ampliado de aceptación de la tecnología. Revista Electrónica de Investigación Educativa, 2l(1), 1. https://doi.org/10.24320/redie.2019.21.e22.1866 